Published in final edited form as:

Mol Nutr Food Res. 2009 May ; 53(5): 529-530. doi:10.1002/mnfr.200990012.

\title{
Arsenic
}

\section{Partha Basu}

Duquesne University, Pittsburgh

Arsenic has a low crustal abundance (1.5-2 ppm), yet it may be present in certain environments in significant concentrations either due to natural occurrence or anthropogenic activity. In nature, arsenic is rarely found as a free element but more commonly as arsenide (e.g., nickel diarse-nide, copper diarsenide), sulfide (e.g., orpiment, i.e. arsenic trisulfide, and realgar, i.e. tet-raarsenic tetrasulfide), or adhered to iron oxides. These forms are insoluble in water, although arsenic oxides are water soluble. Processes such as oxidation of sulfides, release of arsenic from reduction of iron oxides by allochthonous or autochthonous organic matter, exchange of adsorbed arsenate with phosphate, release of arsenic from wood preservatives, organoarsenicals, or release of arsenic from geothermal sources can lead to elevated levels of arsenic in water. Biological activities can enhance such processes, and unlike organic contaminants arsenic cannot be transformed into a completely non-toxic simple compound. Thus, soluble arsenic stays in water systems in a variety of forms and can enter the food chain.

It is the water-soluble forms of inorganic arsenic that have attracted much attention, as inorganic arsenic in a single dose (ca $150 \mathrm{mg}$ ) can be lethal. However, the effects of chronic, low-dose arsenic exposure from drinking water and/or food are more subtle. The conditions leading to arsenicosis are now well documented in certain populations in Southeast Asia. There have been significant efforts to understand the scope of the problem and remediation particularly in West Bengal (India) and Bangladesh. An important component for the success of any remediation is the long-term monitoring of water quality which impacts food and nutrition directly.

There are seven original research articles and one review article in this issue which should provide readers with how arsenic enters into the food chain, how it may exert its toxicity, and to what extent protein expression is influenced by arsenic exposure. Each article published in this issue specifically addresses important aspects such as long-term monitoring of water quality, measurement of arsenic in edible samples, how arsenic interacts with different tissues, and arsenic-induced cellular responses.

The review article by Carbonell-Barrachina et al. describes the endemic arsenic areas, arsenic uptake and accumulated in different classes of food products such as grains, vegetables, fruits and seafood. The article depicts the common practices of food consumption that leads to arsenic exposure and describes both total and inorganic arsenic intake, suggesting both long- and short-term strategies for reducing arsenic intake.

In the first research article, Chakraborti et al. detail a twenty-year study where they analyzed samples from over 140000 tube wells located in different areas in West Ben-gal. About half 
the wells showed arsenic concentrations higher than that recommended by the World Health Organization which can potentially impact nearly 26 million people. However, arsenic concentration decreases with increasing depth. This article underscores the importance of long-term monitoring of water quality. The next three articles report on the speciation of arsenic in seafood and herbs. The last three articles describe the cellular/organelle response to arsenic exposure.

George et al. report on different arsenic species in edible seaweeds, which provides a source of exposure of arsenic to humans. They investigated four different seaweeds, i.e. purple laver, sea lettuce, bull kelp and nori. Using X-ray absorption spectroscopy at As K-edge they identified and quantitated three different forms of arsenic, namely arsenate, an arsenosugar or alkyl arsonium species, and a trivalent arsenic species. A higher level of As(III) species has been detected in sea lettuce and bull kelp. Because As(III) species are recognized to be more toxic and have been difficult to detect this report sheds new light on the origin of potential toxicity in seaweed.

Baeyens et al. report on the determination of total and different forms of arsenic in North Sea fish (19 species) and shellfish (four species), and compared them with those found in fruits and drinks. In general, the total arsenic intake of average Belgian consumers from fish, shellfish, fruit, and soft drinks is $\sim 285 \mu \mathrm{g} / \mathrm{day}$, $95 \%$ of which comes from fish and shellfish; in consumers with higher consumption it may be as high as $\sim 649 \mu \mathrm{g} / \mathrm{day}$. The majority of arsenic is nontoxic and consumption of the toxic forms of arsenic is lower than the recommended tolerable daily intake value.

An interesting aspect of arsenic is its use as a medicine, initially by indigenous people. Medicinal uses of arsenic include treating syphilis, sleeping sickness, and more recently for treating acute promyelocytic leukemia. Cattail (Typha latifolia) is a common food and nutritional ingredient for indigenous people. Lu et al. report on the various arsenic species found in the different parts of the cattail. Using liquid chromatography coupled with mass spectrometry they determined inorganic arsenite, arsenate, dimethylarsinic acid, and monomethylarsonic acid, where the first two species account for $>80 \%$ of the total arsenic. They also found a strong correlation between arsenic and iron in roots and cuticle. They suggest reducing arsenic exposure by removing the skin of the cattail before consumption.

Methylation of arsenic for organoarsenical compounds is well-known and current evidence indicates that methylated species might be more toxic than the parent inorganic species. Among the various modes of action, inhibition of complex nucleotide excision repair is proposed to be of major importance. The paper by Nollen et al. reports that arsenite and its methylated metabolite, monomethylar-sonous acid, attenuated the expression and protein level of a protein believed to be an initiator of nucleotide excision repair, thus providing a molecular mode of action towards carcinogenicity.

In the next paper we move on to an animal model to understand how arsenic can interact at the organismal level by expressing different proteins. Here, Petrick et al. report the toxic effects of in utero arsenic exposure on the developing lung by identifying the altered proteins. They suggest that these alterations may ultimately result in fetal lungs that are 
premature, while also being highly differentiated and branched. This report demonstrates the effects of arsenic on the developing lung.

The article by Zagana et al. evaluates anticancer activity, measuring the arsonoliposomeinduced growth inhibition of four different cancer cell lines with the exception of the breast cancer cells which were minimally affected by arso-noliposomes. This study reveals that all types (leaky $v s$. non leaky) of arsonoliposomes are active towards cancer cells, thus suggesting that they can be used in developing cancer-specific carriers of cytotoxic agents. Furthermore the stability of arsonoliposomes with membrane may not pose problems in developing anticancer drugs.

The success of this issue lies with the authors, reviewers and colleagues. I sincerely thank the authors for their compelling research on arsenic, which demonstrates diverse aspects of arsenic chemistry. My deepest gratitude goes to the reviewers for their unconditional efforts in maintaining the high standard of the journal. My heartfelt appreciation also goes to our colleagues for their enthusiasm which has made this issue possible. And lastly to the readers; your interest in this issue signifies the apprehension that we have about arsenic, and I hope this issue will help develop new ideas for the future.

Sincerely, 